\title{
OFF-STAGNATION POINT TESTING IN PLASMA FACILITY
}

\author{
A. Viladegut and O. Chazot \\ Von Karman Institute (VKI) \\ 72 Chaussée de Waterloo, Rhode-Saint-Genèse 1640, Belgium
}

\begin{abstract}
Reentry space vehicles face extreme conditions of heat flux when interacting with the atmosphere at hypersonic velocities. Stagnation point heat flux is normally used as a reference for Thermal Protection Material (TPS) design; however, many critical phenomena also occur at off-stagnation point. This paper adresses the implementation of an offstagnation point methodology able to duplicate in ground facility the hypersonic boundary layer over a flat plate model. The first analysis using two-dimensional (2D) computational fluid dynamics (CFD) simulations is carried out to understand the limitations of this methodology when applying it in plasma wind tunnel. The results from the testing campaign at VKI Plasmatron are also presented.
\end{abstract}

\section{INTRODUCTION}

Reentry space vehicles face severe heat flux conditions when interacting with the atmosphere at hypersonic velocities. The strong shock wave in front of the vehicle dissociates air molecules into atoms and the gas becomes a mixture of species [1]. A nonequilibrium boundary layer develops surrounding the vehicle after the shock. Within this boundary layer, atom recombination can take place and a diffusive heat flux term should be added to the heat flux balance equation at the wall. Stagnation point is normally considered as the most critical region due to its higher stagnation properties. This is the reason why it is typically used as a reference for TPS design. However, extreme heat flux conditions could also occur at off-stagation point. Thus, it is of interest to develop a methodology able to reproduce these flight environments in ground facility.

The ground simulation of reentry conditions is carried out through the socalled high enthalpy facilities. The lack of capability to reproduce the complete in-flight phenomena in only one facility urges to operate in different types of wind tunnel: shock-tunnel facilities and plasma facilities. While the formers are used to reproduce high Mach numbers for only few milliseconds, the latters can deal with relatively low velocities and very high static enthalpies during

This is an Open Access article distributed under the terms of the Creative Commons Attribution License 4.0, which permits unrestricted use, distribution, and reproduction in any medium, provided the original work is properly cited. 
several minutes. For TPM characterization, two types of plasma facilities are extensively used. Arc-jet plasma facilites, which can reach high power plasma (several megawatt) through an electrical discharge between two electrodes, and Induced Coupled Plasma (ICP) facilities, which are less powerful than arc-jets but they achieve a very pure plasma torch in terms of chemical composition [2]. The experimental campaign that will be presented afterwards is carried out at the ICP-Plasmatron facility located at the VKI.

The purpose of this paper is the implementation of an off-stagnation point methodology able to duplicate the in-flight nonequilibrium boundary layer in plasma facility. Despite, it is normally said that real-scale models should be used for ground testing in order to reproduce many of the hypersonic phenomena, the methodology presented below overcomes this limitation for the boundary layer. Indeed, thickness, gradients, and chemical reactions can be the same as in flight but applied over reduced scale models. In the sections below, the off-stagnation point methodology is presented followed by a brief introduction to the involved facilities at VKI. Then, a CFD assessment focused on its implementation at VKIPlasmatron is described. The results coming from the experimental campaign are also discussed.

\section{THE OFF-STAGNATION POINT METHODOLOGY}

It is known that the heat flux requirements at off-stagnation point are not as demanding as the ones at stagnation point. However, most heat shield designs are still based on this maximum heat flux condition throughout the wall of the vehicle. Since this is a very conservative assumption, it is of interest for a more efficient design to develop a methodology that allows the flight extrapolation at off-stagnation point configuration, thus reducing both costs and weight.

This paper is based on the off-stagnation point duplication methodology presented by Barbante [3]. He states that the following parameters should be reproduced in ground testing for further flight extrapolation: total enthalpy $H_{e}$, density $\rho_{e}$, velocity $U_{e}$, Damköhler number Da (ratio between chemistry and flow characteristic times), and the parameter $x / U_{e}$ where subindex $e$ refers to boundary layer outer edge properties. From the $U_{e}$ and $x / U_{e}$ requirement, the conclusion that a real-scale model sould be introduced in ground facility is imposed. In order to overcome this limitation, one could restrict the application of this methodology to low enough Eckert numbers, i. e., $U_{\infty}^{2} / h_{\infty} \ll 1$. This assumption implies that most of the flow energy is stored in internal energy and, as a consequence, the off-stagnation point boundary layer has to be close enough to the stagnation point of the vehicle. Now, $U_{e}$ does not need to be respected anymore and, what is important, is the static enthalpy duplication $h_{e}$.

A CFD analysis of the methodology is published by Chazot et al. [4] for two different velocity conditions. The nonequilibrium boundary layer is solved over 
a flat plate at different Mach numbers, showing that the supersonic heat flux over the $x$-coordinate is much higher than the subsonic one. By imposing the same equilibrium freestream conditions for all simulations (i. e., $P$ and $h_{e}$ ), it is expected that chemical reactions within both boundary layers are the same. This means that the characteristic time of the chemistry is always respected. Then, considering the variable $x / U_{e}$ as a characteristic time of the flow for offstagnation point configuration, $\mathrm{Da}$ is automatically reproduded. The same publication shows how the heat flux curves for different Mach numbers converge over the $x / U_{e}$ variable, which means the boundary layer development is the same for two different velocities. Indeed, the conservation of the boundary layer thickness $\delta$, if the convective time $x / U_{e}$ is the same, is shown by

$$
\delta=f\left(\frac{x}{U_{e}}\right)=\frac{x}{\sqrt{\operatorname{Re}_{x}}}=\sqrt{\nu \frac{x}{U_{e}}}
$$

where $\operatorname{Re}_{x}$ is the Reynolds number based on $x$ and $\nu$ is the kinematic viscosity.

\section{VKI FACILITIES}

\subsection{The VKI-Plasmatron}

Induced coupled plasma generation uses a cooled quartz tube surrounded by a coil to heat up the gas. If high voltage and high power are applied to the coil, primary currents produce an electromagnetic field coaxial to the coil itself. The time-varying field induces an electromagnetic discharge that leads to dissociation of the gas molecules. Eddy currents within the ionized gas increase the rate of collisions, the gas heats up by Joule effect, and plasma is generated. In addition, to enhance stability and provide a better thermal management of the plasma torch, an annular injection of gas is realized. Once the plasma is generated, it is discharged through a torch into the test chamber.

Plasmatron is able to operate at $1.2 \mathrm{MW}$ of power and $2 \mathrm{kV}$ at $400 \mathrm{kHz}$, to create the plasma, and to expand it into a 2.5-meter long and 1.4-meter diameter test chamber through a 160-millimeter diameter torch. A schematic of the facility is shown in Fig. 1. The control parameters are mainly three: mass flow of gas, static pressure $P$ inside the test chamber, and power applied to the coil. The mass flow is regulated with a rotameter, while $P$ is controlled with vacuum pumps located under the test facility and an atmospheric air by-pass. Despite the power is a control parameter, the one that is normally used to target testing conditions is the stagnation point heat flux which can be measured in real time with the stagnation heat flux probe. References $[5,6]$ are recommended for further details about VKI-Plasmatron facility. 


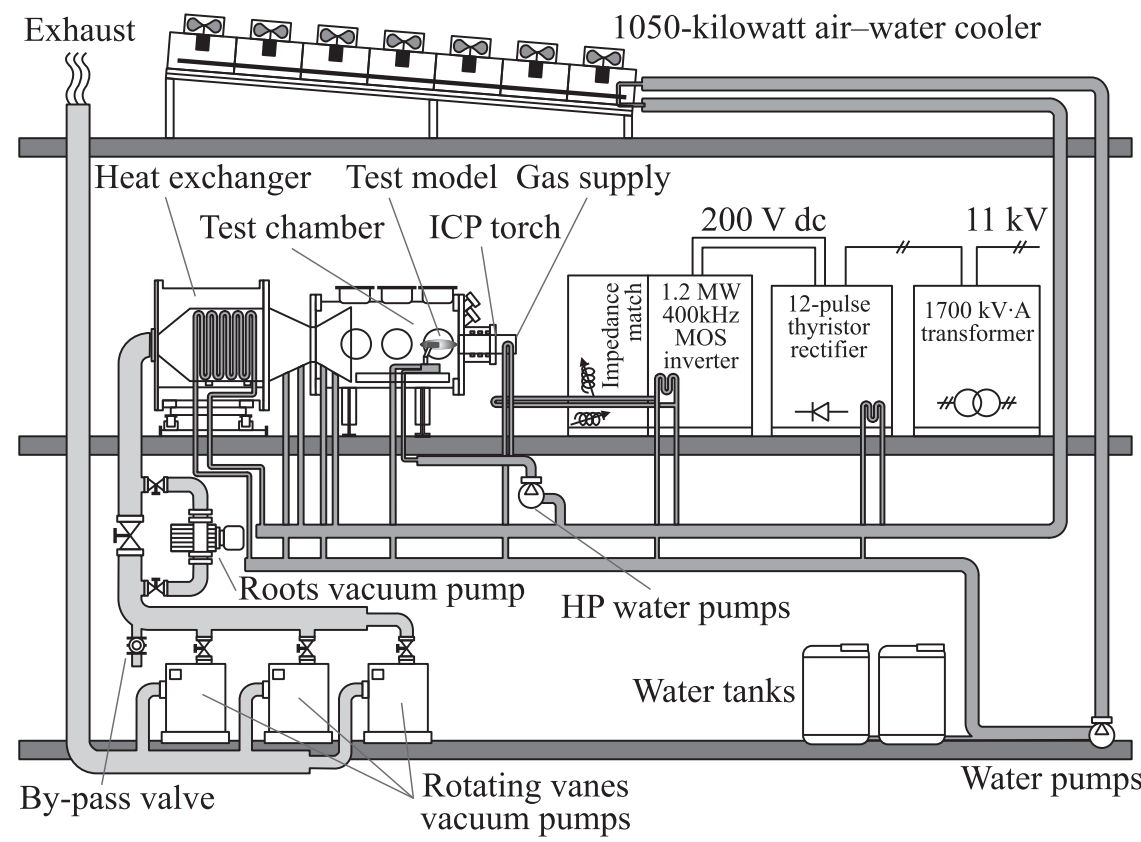

Figure 1 VKI-Plasmatron facility

\subsection{The Flat Plate Model}

The flat plate model $[7,8]$ used during the testing campaign consists of two main elements. On one hand, a copper flat plate holder with two cooling systems containing a water mass flow that keeps the surface temperature at $350 \mathrm{~K}$. On the other hand, a flat plate calorimeter that measures the heat flux at off-stagnation point (Fig. 2). The holder has a groove where the calorimeter (or TPM samples) can be fixed for testing at off-stagnation point configuration.

Eight isolated copper sensors are distributed along the flat plate calorimeter to obtain the heat flux distribution. The insulation issue is very important because energy cannot be lost between sensors or towards the holder. Each sensor is made of a small part of the copper plate that is cooled by a water pipe. It is assumed that all the energy of the copper plate is transmited to the water. Then, type-E thermocouples are used to measure the water temperature $(T)$ increment within each sensor so the heat flux $(\dot{Q})$ distribution can be obtained with

$$
\dot{Q}=\frac{\dot{m}_{\mathrm{H}_{2} \mathrm{O}} c_{p}}{d} \frac{\partial T}{\partial x} .
$$



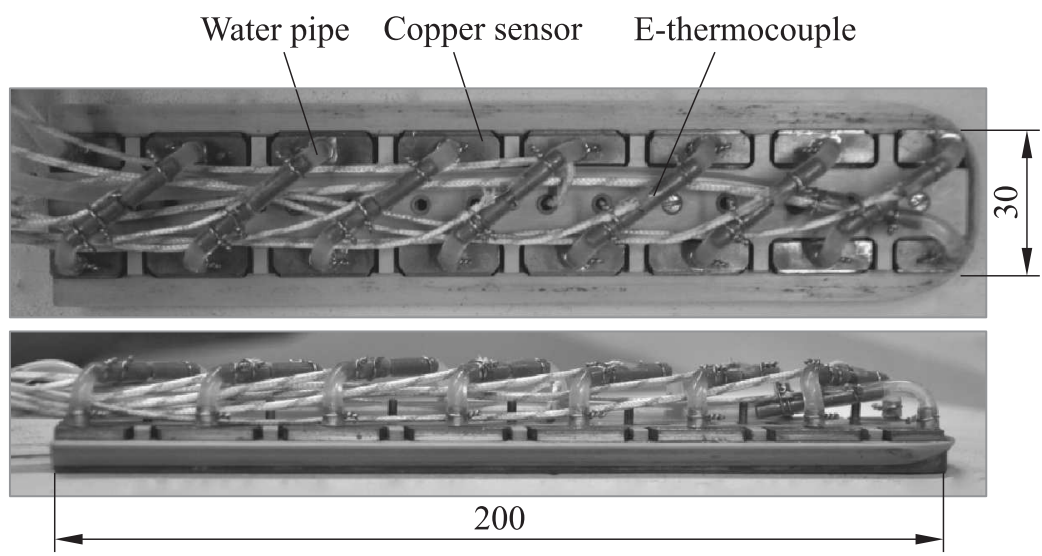

Figure 2 The flat plate calorimeter. Dimensions are in millimeters

Mass flow of water and its specific heat at constant pressure are $\dot{m}_{\mathrm{H}_{2} \mathrm{O}}$ and $c_{p}$, respectively, while $d$ is the width of the sensor $(30 \mathrm{~mm})$.

\section{COMPUTATIONAL FLUID DYNAMICS ANALYSIS}

The implementation of the offstagnation point methology described above at the VKI Plasmatron facility is the major concern of this paper. A 2D CFD analysis trying to reproduce as close as possible the conditions in the facility is carried out before testing. The main goal of the analysis is the applicability of the methodology over a finite flat plate model with a rounded nose. The flat plate model is re-

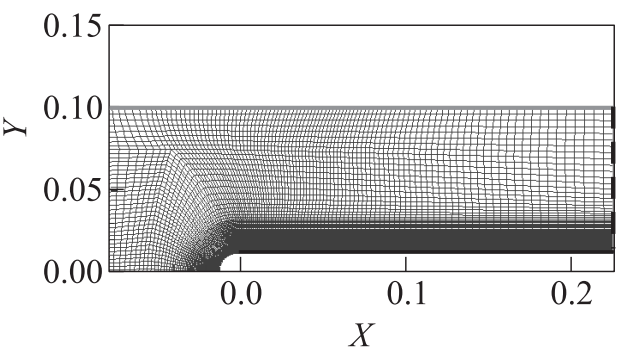

Figure 3 Flat plate model geometry for CFD analysis produced in Fig. 3 for numerical analysis. It is of particular interest to know which variables affect the methodology, especially, the free-stream velocity $U_{\infty}$, pressure $P$, and perpendicular-to-the-flow direction gradients due to nonuniform free-stream conditions of the torch.

For the analysis, the numerical code CFD++ version 11.1 from Metacomp Technologies is used. It solves the nonequilibrium Navier-Stokes equations for a 7-species air model. As the methodology is only applicable to laminar boundary layers, no transition is considered. The software allows the definition of either noncatalytic or full-catalytic conditions at the wall. 


\subsection{Applicability of the Methodology over Finite Flat Plate}

In $[3,4]$, it is proposed that the velocity used to estimate the convective time $x / U_{e}$ along the flat plate should be $U_{e}$. However, a finite flat plate model with a rounded step is used for testing. This means that $U_{e}$ is not constant everywhere along the model and its measurement would be rather difficult. It would be much more convenient to use the free-stream velocity $U_{\infty}$, as it could be verifyied by CFD.

The free-stream conditions used for the analysis are in Table 1. Local thermodynamic equilibrium (LTE) is imposed at the freestream where the species concentrations are specified by VKI Mutation Library. Wall temperature is always set at $350 \mathrm{~K}$. The results can be seen in Fig. 4. The heat flux curves converge along $x / U_{\infty}$ for the same pressure. This means that the obtained boundary layers are equivalent and the methodology is applicable with the free-stream velocity $U_{\infty}$. Pressure is affecting chemical reactions and as a consequence, it defines the resulting heat flux curves. This fact has an important consequence for testing considering that the easiest way to modify $U_{\infty}$ in the Plasmatron is by changing static pressure $P$. Given the results in Fig. $4, U_{\infty}$ should be varied with the mass flow of gas injected at the torch.

\subsection{Reference Enthalpy and Velocity}

It is reasonable to think that the fact of having a gradient in the free-stream properties could significantly influence chemical reactions in the bulk of the boundary layer. However, even if the simplifying assumption of a uniform free stream is rather convenient, it is difficult to achieve in real testing conditions with a plasma torch. Then, the study of the influence of a jet profile discharged on the flat plate becomes a necessity. To analyze this effect, both enthalpy and velocity 


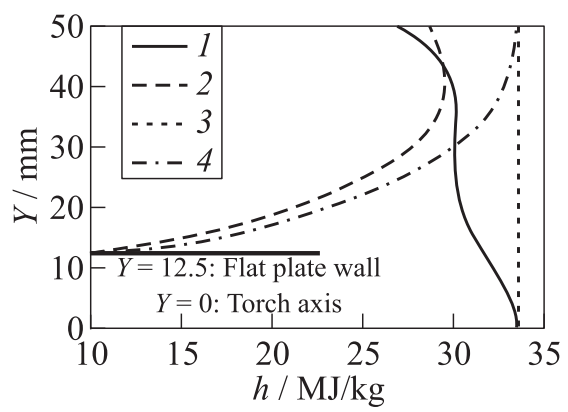

Figure 5 Uniform and jet enthalpy profiles comparison (evaluation at $20 \mathrm{~cm}$ from the leading edge): 1 - jet free stream; 2 - boundary layer from jet at $20 \mathrm{~cm}$ from the leading edge; 3 uniform free stream; and 4 - boundary layer from uniform at $20 \mathrm{~cm}$ from the leading edge

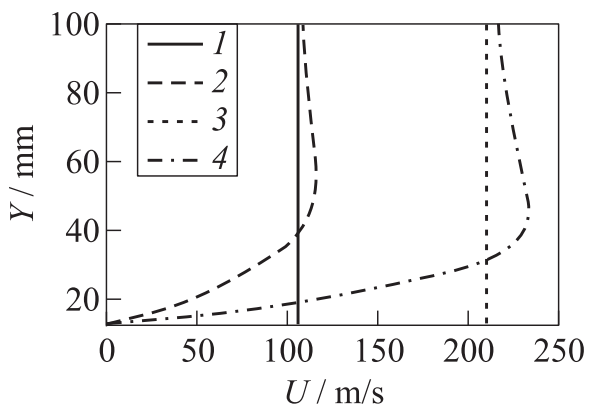

Figure 6 Effect of varying $U_{\infty}$ on $U_{e}$ (evaluation at $20 \mathrm{~cm}$ from the leading edge): $1-U_{\infty}=105 \mathrm{~m} / \mathrm{s} ; 2-$ boudary layer for $U_{\infty}=105 \mathrm{~m} / \mathrm{s} ; 3-U_{\infty}$ $=210 \mathrm{~m} / \mathrm{s}$; and 4 - boundary layer for $U_{\infty}=210 \mathrm{~m} / \mathrm{s}$

profiles are rebuilt from experimental measurements $[9,10]$ across the plasma torch. These profiles, together with species concentrations under equilibrium, have been introduced as input boundary conditions in the simulations. The results are shown in Fig. 5 where the enthalpy profile at $20 \mathrm{~cm}$ from the leading edge is compared to the one obtained under uniform conditions. Free-stream profiles are plotted too. It appears reasonable to define the outer edge as the point where enthalpy reaches its free-stream value. In the profile corresponding to the free-stream jet, the enthalpy at the outer edge is not the same as the one located at the axis of the plasma torch. Then, free-stream enthalpy on the axis should be considered as a reference and not as the one really present at the outer edge. Another conclusion from Fig. 5 is that enthalpy gradients are not significantly different between the two boundary layers. Hence, the assumption of uniform free-stream conditions during the tests should not be a limitation for the methodology. The same conclusions apply at different locations of the flat plate.

In addition, the assurance that a variation in $U_{\infty}$ leads to the same variation at the outer edge $U_{e}$ is required. This has also been verified and it is shown in Fig. 6. Under the same $h_{\infty}$ and $P$ conditions, if $U_{\infty}$ is varied from 210 to $105 \mathrm{~m} / \mathrm{s}$, $U_{e}$ is also halved at $20 \mathrm{~cm}$ from the leading edge of the flat plate model. The same result is checked at different $x$-coordinates over the model.

\section{TESTING CAMPAIGN}

The off-stagnation point methodology has been applied at the VKI-Plasmatron facility. The experimental setup is shown in Fig. 7. A copper calorimeter (called 


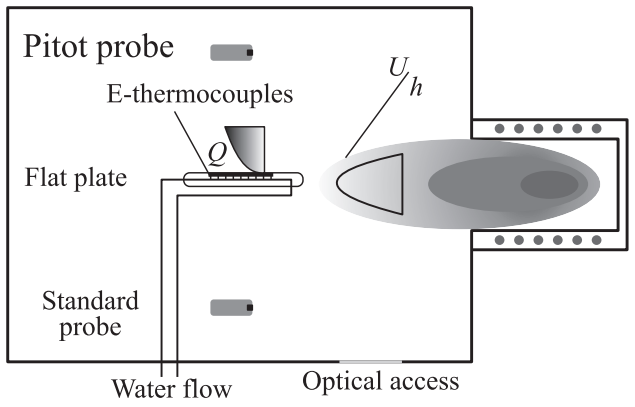

Figure 7 Schematics of VKI-Plasmatron configuration for off-stagnation point testing

Standard Probe at VKI) and a Pitot probe are injected first into the plasma torch to define the testing conditions of heat flux and pressure at stagnation point, respectively. They are used to rebuild the free-stream conditions $U_{\infty}$ and $h_{\infty}$ together with the in-house boundary layer code [11] and ICP simulations [12]. Then, the flat plate model is introduced into the plasma jet and the temperature coming from the themocouoples is stored using the data acquisition system.

The Plasmatron test chamber is equipped with an absolute pressure transducer Memberanovac DM12, Leybold Vacuum, OC Oerlikon Corporation AG, Pfäffikon, Switzerland able to measure static pressure with $\pm 2 \%$ of uncertainty. For the Pitot line, a variable reluctance pressure transducer Validyne DP-15 Validyne Engineering Corp., Northridge, CA, USA is used. Its output is amplified and corrected through a voltage demodulator CD-15 Valydine Engineering Corp., Northridge, CA, USA. Both pressure transducer and demodulator are calibrated by means of a Betz water manometer and their uncertainty is $\pm 10 \%$ due to plasma torch fluctuations. The thermocouples used in the flat plate calorimenter are type- $\mathrm{E}$ with an uncertainty of $\pm 0.2 \mathrm{~K}$.

\subsection{Off-Stagnation Point Results}

The testing procedure described above is repeated for 16 and $8 \mathrm{~g} / \mathrm{s}$ of airplasma for several values of stagnation point heat flux and at constant pressure $(P=1500 \mathrm{~Pa})$. The off-stagnation heat flux distributions with the same static enthalpy are selected. The change of the gas mass flow leads to a variation in the free-stream velocity $U_{\infty}$. The testing conditions considered are presented in Table 2. The off-stagnation point methodology is applied and the results are shown in Fig. 8. Since the difference in $h_{\infty}$ is only 1\%, it is reasonable to assume that chemical composition of the plasma is kept constant between the two cases. The difference in $U_{\infty}$ leads to the conclusion that a 32 percent boundary layer reduction is achieved when passing from 16 to $8 \mathrm{~g} / \mathrm{s}$ air flow. 
Table 2 Testing conditions considered for offstagnation point methodology application

\begin{tabular}{cccc}
\hline$\dot{m}, \mathrm{~g} / \mathrm{s}$ & $h_{e}, \mathrm{MJ} / \mathrm{kg}$ & $U_{\infty}, \mathrm{m} / \mathrm{s}$ & $P, \mathrm{~Pa}$ \\
\hline 16 & 11.73 & 839.93 & 1500 \\
8 & 11.61 & 572.21 & 1500 \\
\hline
\end{tabular}

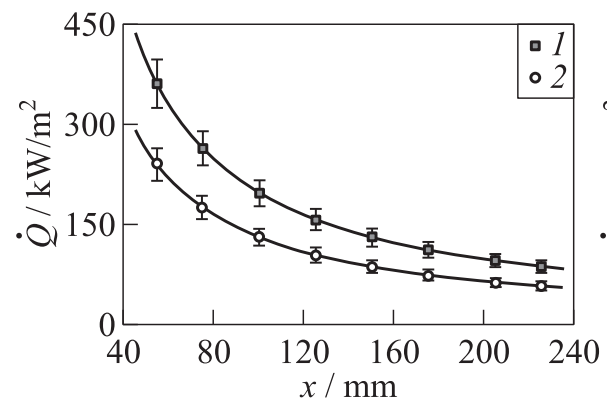

(a)

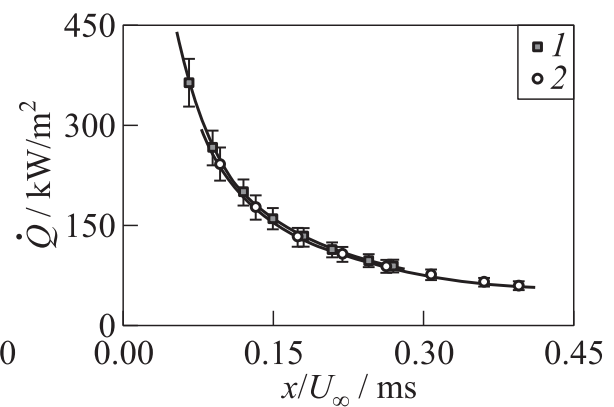

(b)

Figure 8 Off-stagnation point methodology applied at VKI-Plasmatron facility: (a) $\dot{Q}$ over $x$ and $(b) \dot{Q}$ over $x / U_{\infty}: 1-16 \mathrm{~g} / \mathrm{s}: h_{\infty}=11.73 \mathrm{MJ} / \mathrm{kg}$ and $U_{\infty}$ $=839.93 \mathrm{~m} / \mathrm{s} ;$ and $2-8 \mathrm{~g} / \mathrm{s}: h_{\infty}=11.61 \mathrm{MJ} / \mathrm{kg}$ and $U_{\infty}=572.21 \mathrm{~m} / \mathrm{s}$

Uncertainty analysis studies can be found in [6] for the stagnation point heat flux Standard Probe. Same levels of uncertainty are expected at the flat plate calorimeter since both are based on the same measurement principles. Heat flux accuracy is assumed to be $\pm 10 \%$ following

$$
\frac{\delta \dot{Q}}{\dot{Q}}=\sqrt{\left(\frac{\delta \dot{m}}{\dot{m}}\right)^{2}+\left(\frac{\delta c_{p}}{c_{p}}\right)^{2}+\left(\frac{\delta\left(T_{\text {out }}-T_{\text {in }}\right)}{T_{\text {out }}-T_{\text {in }}}\right)^{2}+\left(\frac{\delta A}{A}\right)^{2}}
$$

where 'in' and 'out' refer to input and output conditions at the sensor and $A$ is the corresponding sensing area. Static pressure $P$ and dynamic pressure $P_{\text {dyn }}$ accuracies are $\pm 10 \%$ and $\pm 20 \%$, respectively: the former due to fluctuactions of the vacuum pumps and the latter due to plasma torch instability. Propagation error through the rebuilding boundary layer code leads to \pm 10 percent uncertainty on $h_{\infty}$.

\section{CONCLUDING REMARKS}

An off-stagnation point methodology that overcomes the model size limitation for high enthalpy facilities has been assessed. After a 2D CFD analysis, it is sug- 
gested that VKI-Plasmatron is suitable to apply this methodology. Care should be taken when changing the $U_{\infty}$ since $h_{\infty}$ and $P$ determine chemical reactions in the bulk of the boundary layer. It is shown that applying the methodology with $U_{\infty}$ instead of $U_{e}$ remains acceptable as well as the assumption of uniform free-stream conditions. Finally, the methodology is successfully applied for the Plasmatron testing conditions considered in Table 2 obtaining a 32 percent reduced scale boundary layer over the flat plate model.

\section{REFERENCES}

1. Anderson, Jr., J. D. 1989. Hypersonic and high temperature gas dynamics. AIAA education ser. McGraw Hill.

2. Chazot, O. 2009. Plasma wind tunnel: Design, operation and applications. Von Karman Institute for Fluid Dynamics. Course note 211.

3. Barbante, P. F. 2009. Heat flux duplication between ground facility and hypersonic flight. J. Thermophys. Heat Transfer 23:684-692.

4. Chazot, O., F. Panerai, and V. van der Haegen. 2011. Aerothermochemistry testing for lifting reentry vehicles. 7th European Symposium on Aerothermodynamics Proceedings. Ed. L. Ouwehand. Noordwijk. Netherlands: European Space Agency. Id. 48.

5. Bottin, B., O. Chazot, M. Carbonaro, V. van der Haegen, and S. Paris. 1999. The VKI plasmatron characteristics and performance. RTO-AVT Course on measurement techniques for high enthalpy and plasma flows. Rhode-Saint-Genese, Belgium.

6. Panerai, F. 2011. Aerothermochemistry characterization of thermal protection systems. Von Karman Institute for Fluid Dynamics, Universitá degli Studi di Perugia. Ph.D. Thesis.

7. Vanaerschot, A. 2009-2010. Development of a flat plate probe for off-stagnation testing of space reentry vehicles. Faculteit Ingenieurswetenschappen - Katholieke Universiteit Leuven. Final year project.

8. Braun, A. 2011. Validation of a methodology for off-stagnation point testing. Von Karman Institute; Institute for Space Systems, Stuttgart University. Study Thesis.

9. Baldani, F. 2009. VKI Plasmatron jet investigation for off-stagnation point testing. Von Karman Institute for Fluid Dynamics. Project Report 2009-21.

10. Cipullo, A. 2010. Plasma flow characterization by means of optical emission diagnostics. Von Karman Institute for Fluid Dynamics. Project Report 2010-05.

11. Barbante, P. F. 2001. Accurate and efficient modelling of high temperature nonequilibrium air flows. Von Karman Institute for Fluid Dynamics; Université Libre de Bruxelles. Ph.D. Thesis.

12. Degrez, G., D. Vanden Abeele, P. F. Barbante, and B. Bottin. 2004. Numerical simulation of inductively coupled plasma flows under chemical non-equilibrium. Int. J. Numer. Meth. Heat Fluid Flow 14:538-558. 